\title{
Neural Network Estimators: Application To Ship Position Estimation
}

\author{
D. G. Lainiotis, K. N. Plataniotis \\ Florida Institute of Technology \\ Department of Electrical and Computer Engineering \\ 150 W. University Blvd., Melbourne F1. 32901, USA
}

\begin{abstract}
Ship motion prediction is an important practical problem in design of many ocean systems i.e. fire controls, air craft landing and take off. As such the problem has recelved considerable attention in the past and various attempts for designing estimators via the statistical methodology were made in the past. In this paper the design of the shlp position estimator via neural networks is considered. The ship position estimation is viewed as an adaptive estimation problem for partially unknown systems. New powerful neural estimators based on dynamic recurrent neural networks are applied to the solution of the ship position problem. The new proposed neural estimators, and the state of the art statistical filters, are comparatively evaluated via extensive simulations. The results show that the neural algorithms have excellent performance, achieving significant computational savings due to their massively parallel structure.
\end{abstract}

\section{INTRODUCTION}

The accurate on-line estimation of ship motion is essential to many ship related problems such as ship steering [1], dynamic positioning [2], marine oil exploration [3], off shore platforms, and aircraft landing and take off [4]. Based upon predicted ship motion, the necessary control commands for the control of highly qualified ships like hovercrafts are calculated and generated. Ship motion prediction is also essential when accurate control of position mechanisms for guns or missiles is required. Especially when tracking of a maneuvering target is the objective there is a considerable amount of delay in transmitting information regarding the motion to the position mechanism. It is obvious that accurate predictions are required before an appropriate tracking command can be issued [3],[8],[14].

In the past several studies have been carried out for the solution of the ship position problem, most of them utilizing the Kalman Filter approach, or other least-squares estimation methods [1],[3]. However the design of a statistical estimator like the Kalman Filter requires the definition of a linear model describing the motion of the ship. More specifically, it requires a state-space representation of this motion. In most cases, the model equations are derived from the ship motion spectral density which corresponds to a particular sea-ship condition, the wave excitation input, and a gaussian random noise as driving input. In other words the sea-state magnitude, the ship speed, the ship heading with respect to the waves, and the disturbance pattern of the sea waves are incorporated in the model [5], [6]. The unknown wave excitation input transforms the problem to an adaptive estimation one reducing the effectiveness of the traditional filtering techniques.

Recently, the emerging technology of neural networks [15] has been successfully applied to the solution of the estimation problem [16]-[20]. Given the difficulties the conventional estimators encounter, neural solutions constitute a unique and novel alternative. The trained multilayer perceptrons with their massive parallelism, capability to approximate arbitrary continuous functions, and significant ability, appear to offer a new promising tool in adaptive filtering research.

In this paper the estimation of the ship motion based on a linear state space model that includes the wave-excitation input is proposed. Specifically, the paper is organized as follows. In Section II, the general formulation of the ship motion dynamics is presented. In Section III, new adaptive neural estimators are tested in extensive simulations on the ship motion model. Comparisons with conventional statistical methods in a variety of simulation experiments are also provided. Conclusions are given in Section IV.

\section{SHIP MOTION DYNAMICS}

The motion of the ship can be described by a set of differential equations. As it was mentioned above, the model depends on the sea-state magnitude, the ship speed and the ship heading with respect to the waves. For a rigid ship travelling with a constant forward speed and in the direction which makes an arbitrary angle with regard to sinusoidal waves, its motion can be described by a set of second-order linear differential equations of the form:

$$
a \ddot{q}(t)+b \dot{q}(t)+c q(t)=\xi(t)
$$

where, $q(t)$ represent a vector of surge, sway and heave motions or variations of roll, pitch and yaw orientations, $\xi(t)$ represents the sea-wave excitation, and a,b,c are constants that represents the virtual mass, damping and restoring stiffness, and are determined by the dimensions and shape of the ship. 
The above linear model can be obtained using the power spectral density function under different sea-wave excitation[5]. The most important part of the equation is the representation of the periodic sea-wave excitation. The wave excitation can be approximated by the superposition of sinusoidal waves[6].

$$
\xi(t)=\Sigma A_{i} \cdot \sin \left(\omega_{i} \cdot t+b_{i}\right)
$$

where, $\mathrm{Ai}$ is the amplitude of wave excitation, $\omega_{\mathrm{i}}$ is the frequency, $b_{i}$ the phase of each different wave.

In most of the cases, the amplitude and the frequency of the waves are considered to be time-invariant. When the ship is moving with forward speed, there exist certain relation between the actual wave frequencies, and the frequencies encountered by the ship[6]. Following this relation, the direct influence of the waves on the ship is given as follow

$$
\begin{aligned}
\xi_{a}(l) & =\Sigma A_{i} \cdot \sin \left(\omega_{a i} \cdot l+b_{i}\right) \\
\omega_{c i} & =\omega_{i}-\frac{\omega_{i}^{2}}{8} \cdot v \cdot \cos (x)
\end{aligned}
$$

where, $w_{e}$ is the transformed wave frequency, $v$ is the forward speed of the ship, $x$ is the angle between the ship heading and the wave direction, and $g$ is the standard gravitational acceleration.

Moreover since the energy of each individual wave component rapidly decreases as the frequency of the wave increases, the above expression can be simplified further. Truncating the high frequency components the expression takes the following form:

$$
\xi_{e}(t)=\sum_{i=1}^{N} A_{i} \cdot \sin \left(\omega_{e i} \cdot t+b_{i}\right)
$$

It is reported in numerous studies [1], [5],[6],[7] that a value of 3 for $N$, is sufficient to approximate the wave excitation input, in the case of small ships. After all the approximations and transformations, the equation for the ship motion in the equivalent state space representation can be written as follows:

$$
\dot{x}(t)=\left[\begin{array}{cc}
0 & 1 \\
-\frac{c}{a} & -\frac{b}{a}
\end{array}\right] \cdot x(t)+\left[\begin{array}{l}
0 \\
\frac{1}{a}
\end{array}\right] \cdot \xi_{e}(t)+w(t)
$$

$$
z(k)=[10] \cdot x(k)+v(k)
$$

where, $x(t)$ is the state vector which represents the ship motion. It is a $2 \times 1$ vector defined as follows:

$$
x(t)=\left[\begin{array}{l}
q(t) \\
\dot{q}(t)
\end{array}\right]
$$

$\xi e(t)$ is the truncated wave excitation input, $w(t)$ is the state random noise, $z(k)$ is the measurement vector, $v(k)$ is a random noise which corrupts the measurements.

The position and velocity of the ship are usually measured by on-board sensors. Since all the states are not measurable, and the measurements always contain noise, filters are employed to estimate the actual position of the ship. The objective is to obtain the optimal, in the mean square sense, state estimate $z(k / k)$ of the state $x(k / k)$, given the measurement record, $Z(k)=\{z(1), z(2), ., z(k)\}$.

\section{SHIP POSITION ESTIMATORS}

In the past Kalman Filter based techniques, or other statistical filters have been used in connection with state space models in order to provide meaningful and accurate estimates of the ship motion. If the sea-wave excitation (sea condition) is known in advance, and all other dynamic and statistical specifications of the above model meet the assumptions of the Kalman Filter, then this filter is the optimal estimator and provides the most accurate estimate.

However, it is highly unlikely to know the actual wave excitation input function which directly influences the ship motion. Since the states of the ship are determined by the actual encountered wave excitation input is of great practical importance to derive an estimator that can handle successfully this uncertainty. Due to the uncertainty in the wave excitation input function as given equations (3)-(5) the ship position estimation can be viewed as an adaptive estimation problem where the unknown parameter vector in the model summarizes the uncertainty in the amplitude of the wave excitation and/or the encounter frequencies. It is difficult to estimate the states of the above model using statistical filters when all these parameters must be identified simultaneously without any constraints. Therefore the current methodology is to treat only the corresponding amplitudes as parameters Even in this case, when a mismatch occurs between the actual model and the model used by the designer, the Kalman filter fails.

A more robust statistical filter, namely the Adaptive Lainiotis Filter [8]-[14], was successfully used in this situation. Unlike the Kalman Filter, this new filter, due to its adaptive nature, identifies in real time, the actual model and provides 
the appropriate solutions [8],[14].

Motivated by the recent interest on neural networks application to state estimation problem [16]-[20] a dynamic neural estimator was used in [20] to provide estimates of the current and future ship position. The estimator was an input recurrent multilayer recurrent neural network, trained using back-propagation technique, to provide estimates of the ship position. Since in that analysis the comparative evaluation between neural and the statistical filters was the main objective, the dynamic model of the ship motion was completely known.

The trained multilayer perceptron as state estimator was proved to enjoy certain advantages over the conventional filters derived through the statistical methodology [18],[20]:

- It can handle any assumption or uncertainty concerning the statistics of the actual data generation model. It does not depend on any assumption about the stochastic input. To the contrary the Gaussian nature of the noises in the model, and the independence between the state and the noises, are fundamental assumptions behind any feasible filter derived using statistical methodologies.

- Due to its massive parallel structure and high speed the neural estimator can take full advantage of the new hardware capabilities. That makes the neural and not the statistical estimator the preferable choice for real time signal processing, and automatic control applications.

In this work the wave excitation input is assumed partially or completely unknown. Since the actual model is not available a different approach is used. Several networks are trained independently using different variations of the wave excitation input in the model. Each one of the networks trained with data pairs obtained using different assumptions about the amplitudes and the frequencies in eq. (5). Due to this training methodology each network converges to a different solution. When the training is over, a bank of different neural estimators is available to be applied to the solution of the ship position estimation. In the actual operation phase a nonlinear selection mechanism is used to select at every time instant the neural estimator that provides the best estimate.The new estimator is an extension of the Adaptive Lainiotis Filter to neural networks.

The new adaptive neural estimators are compared with the Adaptive Lainiotis Filter (the most advanced statistical filter for adaptive estimation) in a variety of different uncertainty scenarios.

The following simulation experiments are performed:

\section{A. SIMULATION I}

In this first experiment, the adaptive neural estimator is compared with the ALF. It is supposed for comparison purposes that the dynamic model is partially known. The encounter frequencies in the wave excitation input are known. The only unknown element in this experiment is the actual amplitude in equation (8). The statistical estimator is matched to the actual statistical model of the ship dynamics. The experimental set-up is given below:

1) System Model:

$$
\begin{gathered}
\dot{x}(t)=\left[\begin{array}{cc}
0 & 1 \\
-0.36 & -0.06
\end{array}\right] \cdot x(t)+\left[\begin{array}{l}
0 \\
1
\end{array}\right] \cdot \xi_{e}(t)+w(k) \\
z(k)=\left[\begin{array}{ll}
1 & 0
\end{array}\right] \cdot x(k)+v(k) \\
\xi_{e}(t)=\sum_{i=1}^{3} a_{2 i-1} \cdot \sin \left(\omega_{i} \cdot t\right)+a_{2 i} \cdot \cos \left(\omega_{i} \cdot t\right)
\end{gathered}
$$

where: $a_{2 i-1}=A_{i} \cos \left(b_{i}\right), a_{2 i}=A_{i} \sin \left(b_{i}\right)$, sampling interval, $0.4 \mathrm{sec}$, total measurement time, $60 \mathrm{sec}$, amplitudes of the wave excitation inputs $A_{i}=0.75$, constant excitation frequency $\omega_{i}=\pi / 4$, different phases $b_{i}=2 \pi / 3 ; b_{2}=\pi / 3 ; b_{3}=\pi / 6$, zero mean gaussian plant noise with covariance $Q=0.001$, and white gaussian measurement noise with covariance, $R=0.1$

The initial state $x(0)$ is assumed Gaussian with mean value 0.1 , and variance 1.0

In order to estimate the state of the above model the following estimators had been used in this first experiment:

$$
\text { 2) statistical estimator: Adaptive Lainiotis Filter (ALF) }
$$

The ALF filter employs two Kalman filters matched to the above dynamic and statistical model. The first KF assumes that the amplitude is $A_{i}=0.5$, and the second one uses the value $A_{i}=1.5$. Moreover, the filter starts its recursive cycle assuming initial state estimate, $x(0 / 0)=0.1$, and initial covariance, $P(0 / 0)=1$.

\section{3) adaptive neural estimator}

The adaptive neural estimator uses two trained recurrent neural networks. The first one was trained with data from eq. (8)-(10) assuming that the actual amplitude value was $A_{i}=0.5$. The second one was trained using data generated under the alternative hypothesis about the amplitude, namely $\mathrm{A}_{\mathrm{i}}=1.5$. The detailed configuration for each one of the recurrent networks are summarized below: 
4) input recurrent neural network

network topology:

- two input nodes: the current and the previous measurements are used as input signals.

- two output nodes: the estimates of the system states. The neural network has so many output nodes, as the states of the model.

- two hidden layers with 5-5 hidden nodes respectively

learning parameters:

- learning rate: 0.005 , momentum: 0.2

training procedure:

- backpropagation training algorithm

- the target vector during training is the state vector which is generated running the above model for a given value of the unknown amplitude.

- the network tries to minimize the square error between the current output and the target vector.

- each training set consists of 100 input/output pairs $(z(k)$, $x(k))$.

- the test data record consists of a sequence of data points produced separately from the training record.

- the training procedure is terminated if the training error tolerance is less than 0.01 or if the number of iterations of the training set is more than 5000 .

\section{Observations:}

Since there is no theoretical analysis to justify the performance of the neural estimators Monte Carlo techniques are used to verify the results. The figure of merit used to compare performance is the mean square error averaged over 100 Monte Carlo runs.Namely, the following performance index, is used

$$
M S E=\frac{1}{m c} \cdot \sum_{i=1}^{m c}(x(k)-\hat{x}(k \mid k))^{2}
$$

From the figures it is obvious that the Adaptive Lainiotis Filter as well as the adaptive neural estimator perform very well. In Fig. 2,3 the performances of the estimators in the estimation of the ship position and the errors over 100 Monte Carlo runs, using the same performance index shown above are given. (Fig. 1-4)

\section{B. SIMULATION II}

In a second experiment, a simple recurrent neural network is compared with the Adaptive Lainiotis Filter under a different uncertainty scenario. This time the amplitude is known but the encounter frequencies are assumed unknown. The system model that generates the test data during the operation phase is that of equations (8)-(10). In this simulation study a recur- rent neural network is trained without knowing the exact wave excitation input. However in the operation phase this network is used to provide estimates of the model states. The configuration of the two estimators used in the experiment are summarized below:

\section{1) statistical estimator: Adaptive Lainiotis Filter (ALF)}

The ALF filter employs two Kalman filters matched to the statistics of the actual model. The first KF has complete knowledge of the actual dynamics. Its knows the exact form of the wave excitation input. The second one is simply assumed that there is no wave input in the model. Moreover, the filter starts its recursive cycle assuming initial state estimate, $s(0 / 0)=0.1$, and initial covariance, $P(0 / 0)=1$.

\section{2) input recurrent neural estimator}

network topology:

- two input nodes: the current and the previous measurements are used as input signals.

- two output nodes: the estimates of the system states. The neural network has so many output nodes, as the states of the model.

- two hidden layers with 5-5 hidden nodes respectively.

learning parameters:

- learning rate: 0.005 , momentum: 0.2

training procedure:

- backpropagation training algorithm.

- the target vector during training is a state vector which is generated running the model of eq. (8)-(10) assuming as wave input a random input uniformly distributed over the interval $[-0.5,0.5]$.

- the network tries to minimize the square error between the current output and the target vector.

- each training set consists of 100 input/output pairs $(z(k)$, $\mathbf{x}(\mathbf{k})$ ).

- the test data record consists of a sequence of data points produced separately from the training record using the actual wave excitation pattern.

- the training procedure is terminated if the training error tolerance is less than 0.01 or if the number of iterations of the training set is more than $\mathbf{5 0 0 0}$.

\section{Observations:}

As was expected the Adaptive Lainiotis filter identifies the actual model and uses the matched Kalman filter to estimate the state of the model. The neural estimator also successfully estimate the ship position despite the minimum information used for its training. It must be emphasized that the neural estimator does not require any information about the inputs to the model statistical or deterministic. The performance of the 
statistical filter depends on the information about the possible variations of the unknown elements. If the actual parameter vector is far away from the trajectory values used in the filters bank its performance deteriorates. These possibility will be explored in a final simulation study (Fig. 5-7).

\section{SIMULATION III}

In this final experiment the two adaptive methodologies are compared using a more difficult uncertainty scenario. The filters must provide state estimates without any knowledge about the wave excitation input. This time uncertainty exists in both the amplitude and frequencies. The encounter frequencies are unknown for both the neural and the statistical estimator.The configuration of the two estimators are summarized below:

\section{1) statistical estimator: Adaptive Lainiotis Filter (ALF)}

The ALF filter employs two Kalman filters matched to the above dynamic and statistical model. The first KF assumes that the amplitude is $A_{i}=0.5$, and the second one uses the value $A_{i}=1.5$. However none of the above filters in its bank knows the exact form of the wave input. Both the filters are assume that only the part of the eq.(10) associated with the cosine input is present. The filter starts its recursive cycle assuming initial state estimate, $\hat{x}(0 / 0)=0.1$, and initial covariance, $\mathrm{P}(0 / 0)=1$.

\section{2) adaptive neural estimator}

The adaptive neural estimator uses two trained recurrent neural networks. The first one was trained with data from eq. (8)-(10) assuming that the actual amplitude value was $A_{i}=0.5$. The network did not know the exact form of the input. The network assumed that it was a random input uniformly distributed over the interval $[-0.5,0.5]$. Similar to this the second recurrent net was trained using data generated under the alternative hypothesis about the amplitude, namely $\mathrm{A}_{\mathrm{i}}=1.5$. During its training the wave input was assumed uniformly distributed over the interval $[-1.5,1.5]$. The detailed configuration for each one of the recurrent networks are summarized below:

\section{3) input recurrent neural network}

network topology:

- two input nodes: the current and the previous measurements are used as input signals.

- two output nodes: the estimates of the system states. The neural network has so many output nodes, as the states of the model.

- two hidden layers with 5-5 hidden nodes respectively. learning parameters:
- learning rate: 0.005 , momentum: 0.2 training procedure:

- backpropagation training algorithm.

- the target vector during training is a state vector which is generated running the state space model for a given form of the unknown input.

- the network tries to minimize the square error between the current output and the target vector.

- each training set consists of 100 input/output pairs $(z(k)$, $\mathbf{x}(\mathrm{k}))$.

- the test data record consists of a sequence of data points produced separately from the training record. The actual model as described in simulation I was used to produce the test data record.

- the training procedure is terminated if the training error tolerance is less than 0.01 or if the number of iterations of the training set is more than 5000 .

\section{Observations:}

In this final experiment the neural adaptive estimator performs better than the corresponding statistical one. The state estimates and the estimation errors summarized over 100 Monte Carlo trials are summarized in Fig. 8-11.

Summarizing the results from the above simulation studies the following conclusions can be drawn:

- The neural estimator provides a very reliable solution to the estimation problem The proposed adaptive neural estimator provides accurate and consistent results despite the significantly less information used for its training.

- In more realistic situations where the actual model is not completely known the neural estimator outperforms the conventional estimators. However advanced statistical filters like the Adaptive Lainiotis Filter (ALF) can be used successfully in some cases. On the other hand the adaptive neural estimator was proved the best choice for all the different uncertainty scenarios used in this study.

- The ability of the neural network based estimator to provide accurate solutions to the ship position problem under more realistic assumptions, and its massively parallel structure and high speed, makes it the preferable choice for such real time signal processing applications.

\section{CONCLUSIONS}

The real time ship motion estimation was considered in this paper. The approach taken, was to design a neural network 
based estimator, that can handle more realistic scenarios about the underlying physical model. A comparison with an advanced statistical estimator ALF, was provided. Simulation experiments were carried out in order to assess the performance of the proposed neural estimator. In the ship motion estimation problem the neural network estimator shows excellent performance, though it was derived using minimal information about the dynamics of the model.

\section{REFERENCES}

[1] R.E. Reid, A.K. Tugcu, B.C. Mears, "The use of wave fulter design in Kalman Filter state estimation for the automatic steering problem of tanker in a seaway", IEEE Transactions on Automatic Control, Vol. AC20, No. 7, pp. 917-922, 1984.

[2] T. M. Weiss, T. W. Devries, "Ship motion measurement filter design" IEEE Joumal of Ocean Engineering, Vol. OE-2, No. 4, October 1977, pp. 325-330.

[3] M.S. Triantafyllou, M.Bodson, M. Athans, "Real time estimation of ship motion using Kalman Filtering technique", IEEE Joumal of Ocean Engineering, Vol OE-8, pp. 8-20, January 1983.

[4] M.M. Siolar, B.F. Doolin, "On the feasibility of real time prediction of aircraft carrier motion at sea", IEEE Transactions of Automatic Control, Vol. AC-28, pp. 350-356, March 1983.

[5] R. Bhattacharyya, "Dynamics of Marine Vehicles", Wiley N.Y., 1978.

[6] J.C. Chung, Z. Bien, Y.S. Kim, "A note on ship-motion prediction based on wave-excitation input estimation", IEEE Joumal of Ocean Engineering, Vol. OE-15, No. 3, July 1990, pp. 244-250.

[7] B. Friedland, "Estimating angular velocity from output of rate integrating 8yro", IEEE Transactions on AES-11, July 1975, pp. 551-555.

[8] D.G. Lainiotis et al., "Real time ship motion estimation using Lainiotis Filters", IFAC Workshop, CAMS-'89, Expert Systems in Marine Automation, pp. 293-301, 1989.

[9] D.G. Lainiotis, "Optimal adaptive estimation: Structure and parameter adaptation", IEEE Transactions on Automatic Control, Vol. AC-16, pp. 160-170, April 1971.

[10]D.G. Lainiotis, "Partitioning: A unifying framework for adaptive systems, I-Estimation", Proceedings of IEEE, Vol. 64, pp. 1126-1142, August 1976

[11] D.G. Lainiotis, "Optimal Nonlinear Estimation", International Joumal of Control, Vol. UC-14, pp. 11137-1148, 1971.

[12]D.G. Lainiotis, "Joint detection, estimation and system identification" Inform. Control J.,vol. 19, pp. 75-92,1972.

[13] D.G. Lainiotis, S.K. Katsikas, "Linear and Nonlinear Lainiotis filters Survey", in IFAC Workshop, CAMS-'89, Expen Systems in Marine Automation Proceedings, pp. 280-293, 1989.
[14] D.G. Lainiotis, CJ. Charalampous, P. Gianakopoulos, S. Katsikas, "Real time ship motion estimation", Proceedings Oceans "92, pp. 283-287, Newpon, Rhode Island, 1992.

[15] D.E. Rumelhart, J.L. McClelland, "Parallel Distributed Processing Explorations into the Microstructure of Cognition, Vol. I", M.I.T. Press, 1986.

[16] J.P. De Gruyenaece, H.M. Haffer, "A comparison between Kalman Filters and recurrent neural networks". Proceedings of IJCNN-92, Vol. IV, pp. 247-251, 1992.

[17] A.J. Kanekar, A. Feliachi, "State estimation using artificial neural networks", Proceedings of IEEE Systems and Engineering Conference, Pp. 552-556, 1990.

[18] D.G.Lainiotis,K.N. Plataniotis, D. Menon, C.J. Charalampous, "Heave Compensation via Neural Networks", Intelligent Engineering Systems through Artificial Neural Networks, Vol. 3, pp 143-148, ASME Press, 1993.

[19] D.G.Lainiotis,K.N. Plataniotis, D. Menon, C.J. Charalampous, "Adaptive Heave Compensation via Neural Networks", in IEEE OCEANS'93 Proceedings, Vol. I, pp 243-248, 1993.

[20] D.G.Lainiotis,K.N. Plataniotis, D. Menon, C.J. Charalampous, "Neural Network Application to Ship Position Estimation", in IEEE OCEANS'93 Proceedings, Vol. I, pp 243-248, 1993. 


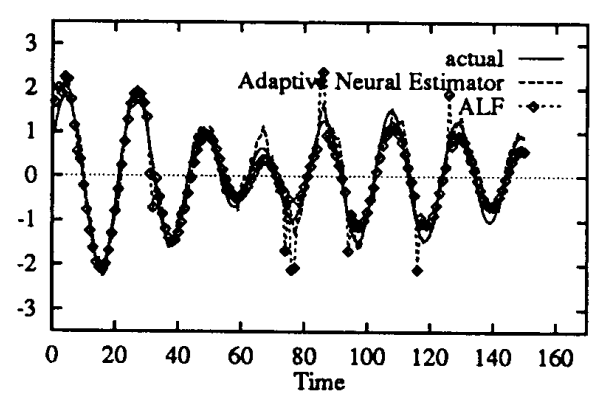

Fig. 1. Ship Position Estimation, Adaptive Neural Network vs. ALF

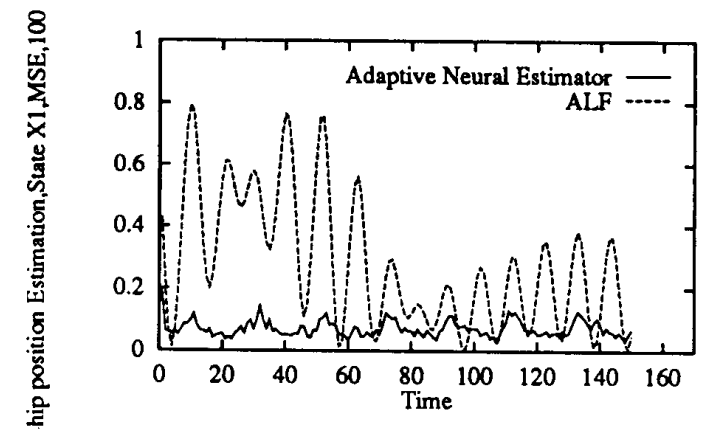

Fig. 2. Ship Position Estimation, Adaptive Neural Networks vs. ALF, MSE Evaluation, 100 MCRs

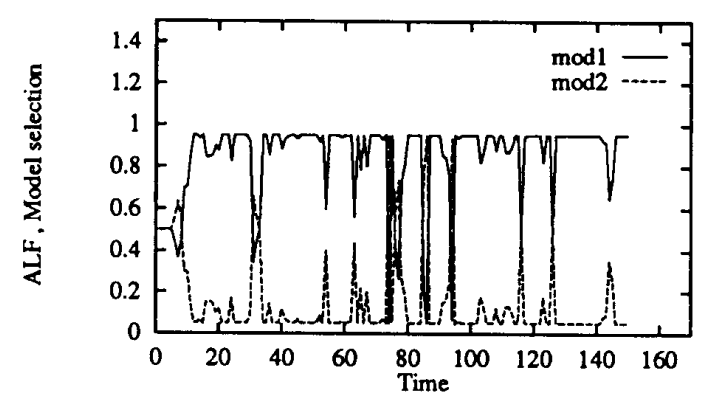

Fig. 3. Ship Position Estimation, ALF, Model Selection

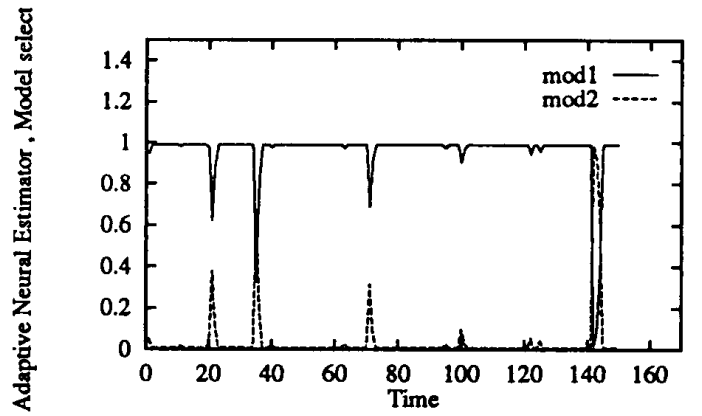

Fig. 4. Ship Position Estimation, Adaptive Neural Estimator, Model Selection

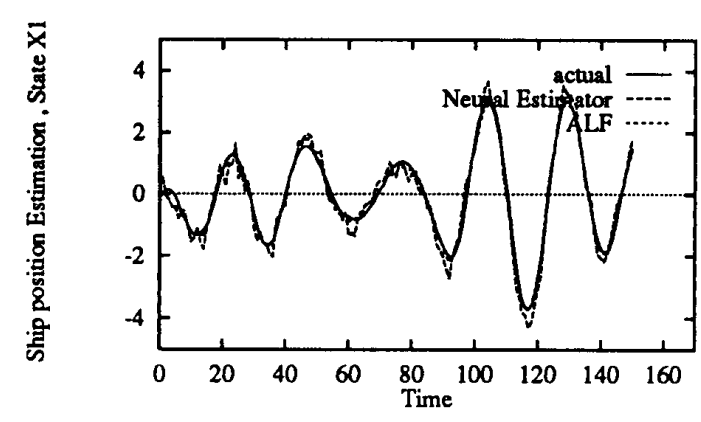

Fig. 5. Ship Position Estimation, Recurrent Neural Network vs. ALF

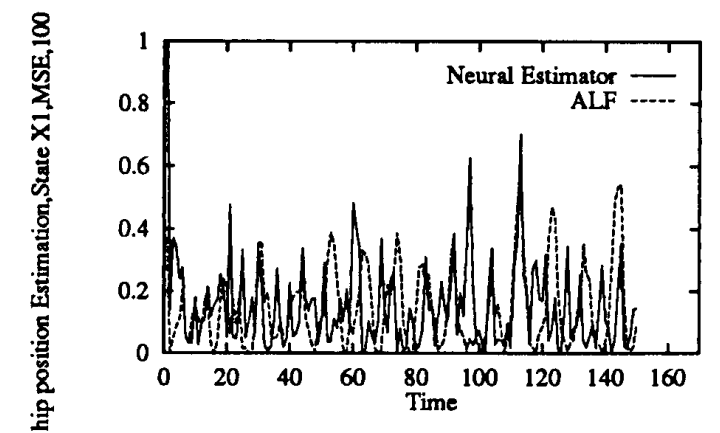

Fig. 6. Ship Position Estimation, Recurrent Neural Networks vs. ALF, MSE Evaluation, $100 \mathrm{MCRs}$ 


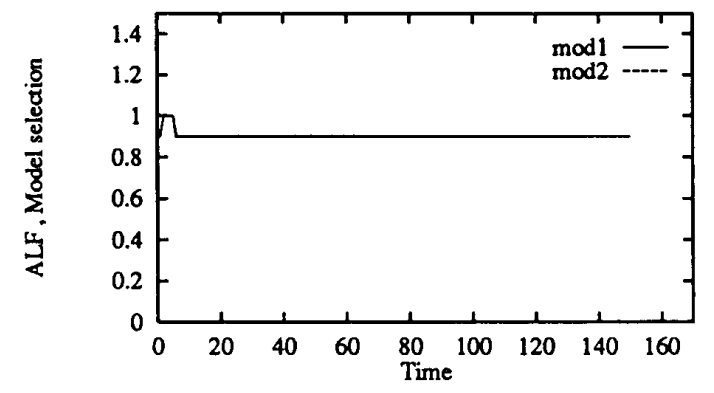

Fig. 7. Ship Position Estimation, ALF, Model Selection

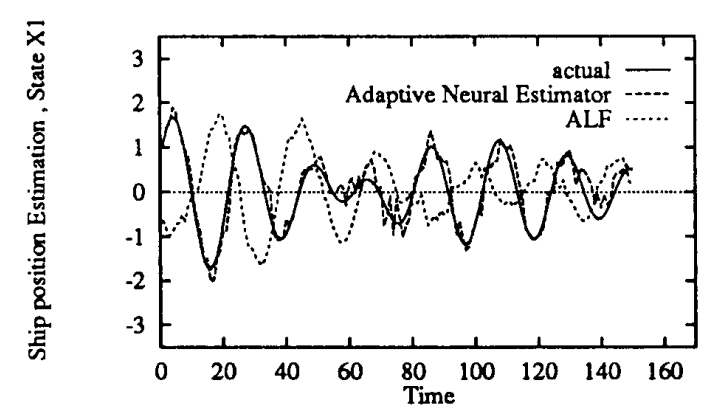

Fig. 8. Ship Position Estimation, Adaptive Neural Network vs. ALF

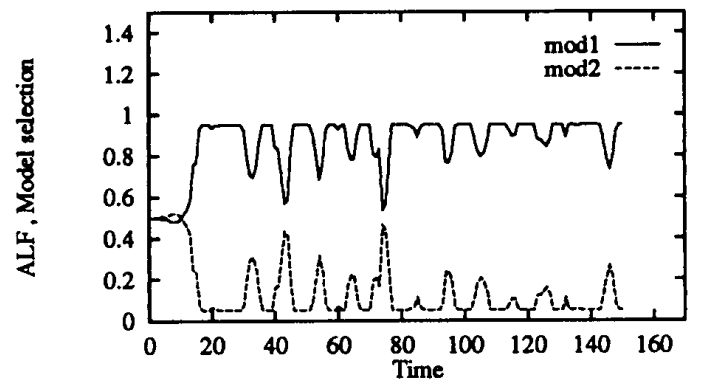

Fig. 10. Ship Position Estimation, ALF, Model Selection

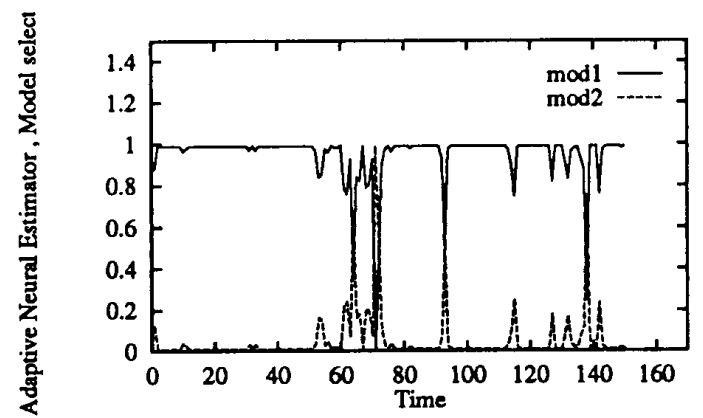

Fig. 11. Ship Position Estimation, Adaptive Neural Estimator, Model Selection

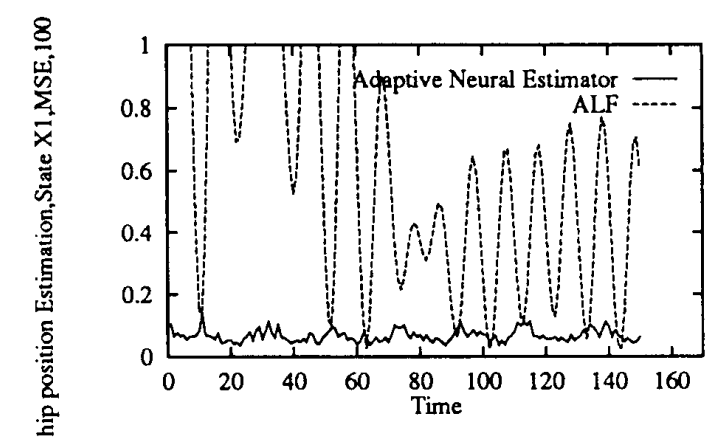

Fig. 9. Ship Position Estimation, Adaptive Neural Networks vs. ALF, MSE Evaluation, $100 \mathrm{MCRs}$ 